\title{
The Formation of the adult human body-chapter 2
}

\section{Introduction}

This is going to be a very long and detailed chapter. Many of the concepts presented will be novel to most people. Considerable background information will be presented in order to make how it will all fit together more easily understood. Many of the concepts that are developed draw heavily from the medical modality known as Acupuncture.

The author has been a practicing Astrologer since 1973. Hippocrates of Kos (460 BC-370BC) stated, "A Physician without a knowledge of Astrology has no right to call himself a Physician". The reason for this statement is that Astrology was developed along the lines of trying to explain both the influence of the cosmos upon life and tried to explain the physical, emotional and mental traits that appeared to be passed from generation to generation. Thus for all practical purposes Astrology was the study of heredity before there was the science of Genetics. The author may testify that Astrology and its spinoff of Astrocartography really do seem to explain the influence of the cosmos upon biological processes. Much rigorous scientific research needs to be done concerning the interface of classical Astrology with genetic processes at the cellular and organismal levels.

Natal chart analysis gives a very good picture of the major factors evident in a person's life. Both physical attributes and mental/ emotional propensities are outlined. Cosmological events in the environment at large are cyclic in nature as are also the biological rhythms within a person. The connection between these two facts is that cosmological events trigger the genetic processes in response to the cosmological fluxuations. A very clear example of this fact is the influence of day length upon the various growth processes of plants, such as leaf budding, flower production, seed maturation and the onset of senescence and leaf dropping. In animals, it is the influence of day length upon the start of mating season, the beginning of hibernation processes and the change in fur color. These changes in behavior and physical characteristics are orchestrated by genetic processes that are cued today length. However, day length is but only 1 of the many cues for the timing of the biological process that are orchestrated by rhythmic changes in genetic expression.

The astrological matrix presented by an accurate natal chart will show both how all of the cosmological influences effect day to day genetic expression that is the key to physiology and behavior at any point in time and also the results of the blended genetic determiners of adult physical, emotional and mental characteristics. Thus, if you understand a person's natal chart, you understand why and how that person functions. Many people try to use Astrology to predict the future. This is wish full thinking upon their part. These people fail to realize that yes cosmological events do set the background for behavioral expression but that the freewill of a person and social interactions determine the actual outcome of the influences afforded by background events, i.e., you can lead a horse to water but you cannot make it drink!

So! Apparently genetic processes evolved to respond to the zeitgebers (A zeitgeber is any external or environmental cue that entrains, or synchronizes, an organism's biological rhythms to the Earth's 24-hour light/dark cycle and 12 month cycle) and the date,

\author{
Volume I Issue 3 - 2015
}

\author{
Edward F Block \\ Block Institute for Astrobiological Studies, USA \\ Correspondence: Edward F Block, Block Institute for \\ Astrobiological Studies, 375 Dula Springs Road,Weaverville, \\ North Carolina, USA, Email efblockiv@yahoo.com \\ Received: June 09, 2015 | Published: July 24, 2015
}

place and timing of fertilization as well as the date, place and time of birth determine the genetic expression that leads from fertilized ovum to fetus to baby to teen to adult. Everything that you are has been determined by the sets of genes that you received from both of your parents. The sets of genes that you received are the result of millions of years of evolution within the environment of the biosphere of the Earth that is under the influence of the cosmos. Much of the information imparted below may be found in bits and pieces online. ${ }^{1.2}$

However, it is in this work that all the scattered bits and pieces have been brought together into a comprehensive whole. The author has utilized and presented here that information so as to make more understandable the intent of this work and not to have the reader jump around following links to other websites.

\section{Fertilization and fetal development}

The formation of the body occurs from the development of the original haploid egg and sperm uniting to form a diploid zygote. The development of the fetus from one individual cell is a very carefully orchestrated series of events that occurs in roughly 270 days. The study of developmental biology is a very interesting source of information concerning this very carefully orchestrated events. ${ }^{3}$

\section{Ovum encoding}

Please remember that the date, time and the location on the Earth upon fertilization of the ovum seems to influence how the sets of inherited genes interact with each other during the process of fetal development. This includes the factors related to the overall physiology of the Mother during her lifetime and just subsequent to the act of fertilization. Environmental influences upon the state of the ova within her ovaries and possible gene expression is called epigenetics (Hiroyuki S. \& Yasuhisa M. (February 2008) Epigenetic events in mammalian germ-cell development: reprogramming and beyond. Nature Reviews Genetics 9, 129-140 | doi:10.1038/nrg2295). Epigenetics refers to changes in gene expression due to mechanisms other than changes in DNA sequence, i.e. as RNA regulator gene expression. And the epigenetic profile of germ cells, which is defined by regulated modifications of DNA expression capability, changes dynamically during their cellular development. Many of the 
changes are associated with the acquisition of the capacity to support post-fertilization development. This is vital for the generation and development of the meridian system in the fetus. Hence, the capacity for this development is expressed in the genetic complement of every ovum in preparation for fertilization. The cellular architecture of the cell is organized by the centriole via microtubules. The cellular membrane is connected to this internal architecture and waiting for the injection of the genetic material and centriole by the fertilizing sperm cell. It is the point of penetration by the sperm cell that initiates a series of events those results in the first cleavage of the fertilized cell into what becomes the right and left sides of the resulting fetus. Thus, the first meridians to come into existence are the conception and governing vessels on either side of the taiji pole (point of DNA injection). The taiji pole is the mouth/anus axis. The second cleavage is in what is now the horizontal plane and yields 4 cells that determine ectoderm (yang) and endoderm (yin). The 2 cells above become the ectoderm and the 2 cells below become the endoderm. The electrical polarity and field of energy in the polar axis is related to the Original or Yuan Qi from which the Conception and Governing Vessels form the seas of Yin and Yang energy during the first cellular division. The Yin and Yang Heel Vessels are also established, forming the future left and right sides. The exterior of the egg is determined by the Yang Linking Vessels. The interior of the egg by the Yin Linking Vessels. What occurs next is a very complicated process that goes through a blastodisc stage that will become the actual fetus. These meridians are the first to form in what is going to be the fetus, and are carriers of Yuan Qi, the ancestral energy which corresponds to our genetic inheritance. The gene sets of allels (the two forms of the same gene on a chromosome) that are responsible for the overall morhogenic field of the body work in conjunction with and through these 8 fundamental human body-field determiners. The specific meridians are: (1) Du Mai (Governing Vessel), (2) Ren Mai (Conception Vessel), (3) Chong Mai (Penetrating or Thrusting Vessel), (4) Dai Mai (Belt Channel), (5) Yang Chiao Mai (Yang Motility Channel), (6) Yin Chaio Mai (Yin Motility Channel), (7) Yang Wei Mai (Yang Regulating Channel), and (8) Yin Wei Mai (Yin Regulating Channel). These 8 prenatal vessels function continuosly throughout the life of the individual and play a vital role in healing. More upon this subject in a later chapter.

\section{Fetal development}

The 8 prenatal vessels (also known as the extraordinary vessels) are said to initially form a vortex of energy in the center of the embryo body, the Taiji Pole, that will be located in the area between what will become the kidneys. The Taiji Pole and the Chong Mai are at the center of this vortex and will form the Sea of 5 Yin and 6 Yang Organs, the Sea of 12 Primary Channels and the Sea of Blood. From this center, Qi and Blood are distributed at the energetic level through small channels or rivers of energy (meridians or nadis). This energy vortex may be hypothesized to create the initial morphogenic field pattern for the growth of the physical form of the developing embryo via functional domain compartmentalization created by cellular junctions between various connective tissue cells. These compartmentalizations create the spheres of influence within which the various organ systems develop and also create the channels for blood flow vessel growth and nerve axon growth that will eventually link the various discrete regions with the whole. As the fetus continues to grow, so do the 20 channels. Both meridian development and blood vessel/nerve axon growth occur congruently. The nine Yang channels begin to flow out of the Governing Channel [Sea of Yang] (Bladder, Gallbladder, Stomach, Small Intestine, San Jiao [Triple Burner], Large Intestine, Yang Heel, Yang Linking and Belt) as the nine Yin Channels begin to flow out of the Conception Vessel [Sea of Yin] (Kidney, Liver, Spleen, Heart, Lung, Pericardium, Yin Heel, Yin Linking and Thrusting). The result is known as the meridian/collaterals system with the 12 main meridians, the 8 prenatal meridians, the 12 divergent meridians, the 15 collaterals, the 12 muscle regions and the 12 cutaneous regions. These vessels of energy travel and distribution are what keep the physical structures coordinated in physiology and anatomy within the overall morphogenic field. The key is that all the genetic expression within the individual cells of the body are regulated by the energy system. The particular gene expression of the cell is tailored by the meridian/ organ system for the development of the various tissues and organs.

All individuals start fetal life as females. In females, it appears that 1 of the $\mathrm{X}$ chromosomes is turned off and becomes essentially inert. Epigenetic factors seem to have a role in determining which $\mathrm{X}$ chromosome is turned off. In males, the $\mathrm{X}$ chromosome plays the dominant role in gene expression as a whole. However, the $\mathrm{Y}$ chromosome asserts a role in the changing of the body pattern from female to male. Gene allels on the Y chromosome produce compounds that "masculinize" the developing brain. The male masculinized brain responds differently to circulating testosterone than does a female. Also, the primordial ovaries become testes and descend into the scrotum that is formed by the 2 lips of the fetal vagina. The fetal clitoral organ develops into the penis. In females, the uterus forms and in males the prostate forms.

\section{Parturition}

Unless there is a reason for a birthing intervention, the fetus determines the time of the onset of the birthing process. Please remember that the date, time and place of birth appear to have a major role in the determination of the particular sets of gene allels expressed by means of epigenetic factors. The birthing process starts about 270 days post fertilization. When the baby is externalized, the mother still plays a major role in genetic entrainment through the influence of her body-field. This ceases at some point in the teen years of the individual and complete genetic independence by the teen will then ensue.

\section{Acknowledgments}

None.

\section{Conflict of interest}

The authors declare that there is no conflict of interest.

\section{Funding}

None.

\section{References}

1. Block EF. Acupuncture as Informational Medicine. Journal of Informational Medicine. 2010.

2. Block EF. The Role of Coherent Resonance in Human Affairs Part TwoAffairs of Consciousness. Journal of Informational Medicine. 2011.

3. Developmental biology 